\title{
Human T-cell Leukemia Virus Oncoprotein Tax Represses Nuclear Receptor-Dependent Transcription by Targeting Coactivator TAX1BP1
}

\author{
King-Tung Chin ${ }^{1}$, Abel C. S. Chun ${ }^{1}$, Yick-Pang Ching ${ }^{1,2}$, Kuan-Teh Jeang ${ }^{3}$, \\ and Dong-Yan Jin ${ }^{1}$ \\ Departments of ${ }^{1}$ Biochemistry and ${ }^{2}$ Pathology, The University of Hong Kong, 21 Sassoon \\ Road, Pokfulam, Hong Kong; and ${ }^{3}$ Laboratory of Molecular Microbiology, National \\ Institute of Allergy and Infectious Diseases, 9000 Rockville Pike, Bethesda, MD 20892- \\ 0460, USA
}

Running title: HTLV-1 Tax targets TAX1BP1 to repress NR signaling

Note: D.-Y. Jin is a Leukemia and Lymphoma Society Scholar.

Requests for reprints: Dong-Yan Jin, Department of Biochemistry, The University of Hong Kong, 3/F Laboratory Block, Faculty of Medicine Building, 21 Sassoon Road, Pokfulam, Hong Kong. Phone: +852-2819-9491; Fax: +852-2855-1254; E-mail: dyjin@hkucc.hku.hk.

Total text word count: 4,932 


\begin{abstract}
Human T-cell leukemia virus type 1 oncoprotein Tax is a transcriptional regulator that interacts with a large number of host cell factors. Here, we report the novel characterization of the interaction of Tax with a human cell protein named TAX1BP1. We show that TAX1BP1 is a nuclear receptor co-activator that forms a complex with glucocorticoid receptor. TAX1BP1 and Tax co-localize into intranuclear speckles that partially overlap with but are not identical to the PML oncogenic domains. Tax binds TAX1BP1 directly, induces the dissociation of TAX1BP1 from the glucocorticoid receptorcontaining protein complex, and represses TAX1BP1's co-activator function. Genetic knockout of Tax1bp1 in mice abrogates the influence of Tax on the activation of nuclear receptors. We propose that Tax-TAX1BP1 interaction mechanistically explains the previously reported repression of nuclear receptor activity by Tax.
\end{abstract}




\section{Introduction}

Human T-cell leukemia virus type 1 (HTLV-1) was the first retrovirus shown to be associated with a human disease, adult T cell leukemia (ATL). ATL is an aggressive neoplasm; however, its induction by HTLV-1 is a slow process which takes 20 to 40 years and apparently requires the consummation of several discrete events in the virus-infected cell (1).

The HTLV-1 Tax oncoprotein can immortalize T cells and transforms rat fibroblasts (2). Tax is a transcriptional factor, which targets the HTLV-1 long terminal repeats (LTR), heterologous viral promoters, and a variety of cellular genes (3) Tax acts potently through Tax-responsive (TRE) DNA elements on the LTR which are also cAMP-responsive motifs recognized by the cellular transcription factor CREB; Tax can also activate NFkB (3). In various settings, Tax has been found to repress the expression of cellular genes including DNA polymerase $\beta(4), \mathrm{p} 53(5,6)$, nuclear receptors (7), cyclin A (8), c-Myb (9), MyoD (10) and transforming growth factor $\beta$ (11).

Transcriptional activation of gene expression exerted by Tax through either the CREB or the NFkB pathway has been well studied. Accordingly, we understand that Tax functions as a homodimer $(12,13)$, which docks with dimeric CREB in a ternary complex onto the HTLV-1 21-bp TRE motifs (14). Optimal activation of the HTLV-1 LTR by Tax requires the core HTLV-1 TATAA promoter, CREB and the TRE (15). Separately, Tax activation of NFkB is thought to occur through direct interaction with $\mathrm{p} 50 / \mathrm{p} 52$, IкB and IKK $\gamma$ (3). Moreover, Tax can also engage transcriptional co-activators such as CREB-binding protein (CBP), p300, P/CAF and TORC1/2/3 (16-18).

While we know much about how Tax activates transcription, we know considerably less about how it represses gene expression. Several ideas have been forwarded as to how Tax may repress the expression of cellular genes. For example, Tax has been reported to suppress the transcription of the 
DNA polymerase $\beta$ gene via direct inhibition of basic helix-loop-helix factors (19). Tax attenuates cyclin A expression through sequestration of CREB/ATF (8), and Tax suppresses transforming growth factor $\beta$ indirectly by activating the NFKB and c-Jun signaling pathways, respectively ( 9 , 11). Finally, Tax physically binds general transcription co-activators such as CBP, p300 and P/CAF $(10,20)$; its ability to inactivate the function of the p53 tumor suppressor and other targets is thought to be mediated at least partially through binding to $\mathrm{CBP}$ and/or p300 $(9,10,20)$.

Nuclear receptors (NRs) are a large family of ligand-activated transcription factors that regulate gene expression in response to steroids, retinoids and other signaling molecules (21). These transcription factors play an important role in regulating development, differentiation, metabolism and cell proliferation. Dysregulation of nuclear factor-dependent transcription leads to various diseases including cancer (22). A few years ago, Doucas and Evans reported that the HTLV-1 Tax protein is a general repressor of the transcriptional capacity of several NRs including glucocorticoid receptor (GR) (7). To date, it has remained unclear how Tax mechanistically suppresses the activity of NRs. Here we show that TAX1BP1, a human cell encoded Tax-binding protein identified by us (23), is closely related to coiled-coil transcriptional coactivator (CoCoA), a component of the p160 NR coactivator complex $(24,25)$. We further show that TAX1BP1 is a transcriptional coactivator for NR, and that Tax represses NR function through binding to TAX1BP1. 


\section{Materials and Methods}

Plasmids. Human TAX1BP1 cDNA containing a complete coding region (GenBank U33821) was derived from an EST clone (ATCC: 104248) obtained from the American Type Culture Collection. Expression plasmids pSVBP1 and pHABP1 for full-length TAX1BP1 were derived from previously described SV40 promoter-driven expression vectors $\mathrm{pSV}$ and $\mathrm{pHA}$ (26, 27). Plasmid pGSTBP1 contains TAX1BP1 cDNA inserted into pGEX4T3 (Amersham). TAX1BP1, Tax and the activation domain of VP16 fused to Gal4 DNA binding domain (amino acids 1-147) were designated GalBP1, GalTax and GalVP16, respectively. Mammalian expression plasmid pGALBP1 expressing GalBP1 was derived from pM (Clontech). Truncated versions of TAX1BP1 were constructed by PCR and the sequences of all mutants were determined to confirm that no undesired changes were introduced. Mouse Tax1bp1 cDNA (IMAGE: 1480596) in an SV40 promoter-driven expression vector pME18S-FL3 was purchased from the American Type Culture Collection.

Expression plasmids for Tax, GalTax and polyhistidine-tagged Tax (His-Tax) have been described elsewhere $(15,18)$. Expression plasmid for GalVP16 was from Clontech.

Chloramphenicol acetyltransferase (CAT) reporter plasmid pG5CAT containing five copies of Gal4-binding sites was from Clontech. Luciferase reporter plasmid pLTRLuc under the control of the HTLV-1 LTR has been described elsewhere (15). Reporter plasmids pкBLuc, pSRELuc and pCRELuc were from Stratagene. pGRELuc was from Clontech.

Expression plasmid pCDNArGR (28) for rat glucocorticoid receptor (GR) was kindly provided by Dr. Keith Yamamoto (University of California, San Francisco, California, USA). Expression plasmid pSG5TIF2 (29) for human glucocorticoid receptor-interacting protein 1 (GRIP1), also known as transcriptional intermediary factor 2 (TIF2), was a gift from Dr. Hinrich 
Gronemeyer (Institut de Génétique et de Biologie Moléculaire et Cellulaire, Illkirch Cedex, France).

Antibodies. Polyclonal anti-TAX1BP1 antibody $\alpha-151 \mathrm{C}$ was raised in rabbits against a keyhole limpet hemocyanin-conjugated synthetic peptide which corresponds to the C-terminal 21 residues (amino acids 725-747) of human TAX1BP1. Likewise, $\alpha-151 \mathrm{~A}$ was against a conjugated peptide corresponding to amino acid residues 537-556 of human TAX1BP1. $\alpha-151 \mathrm{C}$ and $\alpha-151 \mathrm{~A}$ antisera were purified through HiTrap Sepharose columns (Amersham) activated by $N$-hydroxysuccinimide and coupled to immunizing peptides.

Mouse monoclonal anti-Tax antibody (clone 168A51-42) and rabbit polyclonal antiserum against Tax have been described $(26,30)$. Mouse monoclonal anti- $\alpha$-tubulin (clone B-5-1-2) was from Sigma. Mouse monoclonal anti-HA (clone F-7), anti-PML (clone PG-M3) and anti-GR (clone FiGR) antibodies as well as rabbit polyclonal antiserum H300 against GR were from Santa-Cruz. Rabbit polyclonal antiserum against Gal4 activation domain (AD) was from Upstate.

GST pull-down assay. Recombinant proteins GST, GST-TAX1BP1 and His-Tax were expressed from Escherichia coli and purified as previously described (30). Briefly, equal amounts of His-Tax were loaded onto GST- or GST-TAX1BP1-bound glutathione-Sepharose (Amersham) and flow-through fractions were collected. The resins were washed with three changes of buffer A (20 mM HEPES-KOH, pH 7.9, $20 \mathrm{mM} \mathrm{KCl,} 1 \mathrm{mM} \mathrm{MgCl}_{2}, 17 \%$ glycerol, and $2 \mathrm{mM}$ dithiothreitol) and eluted with buffer A containing $0.5 \mathrm{M} \mathrm{KCl}$. The eluates were precipitated with trichloroacetic acid, solubilized and then analyzed by SDS-PAGE and Western blotting.

Protein analysis. Yeast two-hybrid assays based on Gal4 DNA-binding and activation domain fusions were carried out in strains SFY526, CG1945 and Y187 as previously described 
$(13,30)$. Experimental procedures for immunoprecipitation and Western blotting have also been detailed elsewhere $(27,31)$.

Confocal microscopy. Laser scanning confocal microscopy was performed as previously described $(27,32)$. Dual immunofluorescent detection was achieved with primary antibodies from different species and pre-adsorbed species-specific secondary antibodies conjugated to Cy5 or fluorescein (Zymed). Co-localization of proteins was based on multicolor imaging and was analyzed with Zeiss LSM510 software.

Reporter assay. HeLa cells and mouse embryonic fibroblasts (MEF) were grown in Dulbecco's modified Eagle's medium. HeLa cells were transfected by calcium phosphate precipitation method for CAT assay $(13,26)$. HeLa and MEF cells were transfected by LipofectAMINE $2000^{\mathrm{TM}}$ reagent (Invitrogen) for luciferase assay (33). Jurkat, JPX9 and MT4 cells were cultured in RPMI 1640 medium and transfected by electroporation (18).

CAT activity was assayed by thin-layer chromatography as previously described $(13,31)$. Protein concentration of clarified lysates was determined by Bradford reagent (Bio-Rad) and equal amounts of proteins were added to the CAT reaction. Transfection efficiencies were normalized with a pSV- $\beta$-Gal control plasmid (Promega) expressing $\beta$-galactosidase.

Luciferase activity was measured as described (33) using the Dual-Luciferase ${ }^{\circledR}$ reagents (Promega). Transfection efficiencies were normalized with a pRL-TK control plasmid (Promega) expressing Renilla luciferase.

Generation of Tax1bp1 ${ }^{+/-}$and Tax1bp1 ${ }^{-/-}$mice. The Taxlbpl gene is located on chromosome 6. A 12kb EcoRI-BamHI fragment which contains exon 16 and 17 was used for generation of targeting plasmid. The thymidine kinase $(T K)$ and the neomycin (neo) genes were used as selectable markers. In engineering the knock out mice, exon 17 of Taxlbpl was replaced 
by neo. Confirmation of successful knockout was based on PCR and Western blotting. Details on knock out and in breeding of Tax $1 \mathrm{bp} 1^{-/-}$animals are presented elsewhere (Iha et al., in preparation). 


\section{Results}

Interaction of TAX1BP1 and Tax. We screened a human cDNA library for Tax-binding proteins using yeast two-hybrid technology. One Tax-binding cDNA was initially designated TXBP151 (GenBank U33821) and was subsequently named by the Gene Nomenclature Committee of the Human Genome Organization as "Tax1-binding protein 1 (TAX1BP1)". TAX1BP1 has 747 amino acid residues, and its molecular mass is predicted to be $86.5 \mathrm{kDa}$. We previously reported that TAX1BP1 interacts with A20, which is an inhibitor of apoptosis and NFKB signaling (23). Moreover, others have identified TAX1BP1 as T6BP, a TRAF6-binding protein (34).

TAX1BP1 contains an N-terminal domain (residues 1-150) homologous to nuclear dot protein 52 (NDP52), whose function remains elusive and controversial (35). The central region (residues 150-600) of TAX1BP1 is predicted to form a coiled-coil structure (36). The C-terminal part (residues 668-747) of TAX1BP1 harbors zinc finger motifs (Fig. 1A). Interestingly, the full TAX1BP1 protein also shares $27 \%$ identity and $45 \%$ similarity with a newly-identified coiledcoil transcriptional coactivator $\operatorname{CoCoA}(24,25)$. We found that the TAX1BP1 mRNA is ubiquitously expressed in various human tissues (data not shown).

As a first step towards understanding TAX1BP1 and its interaction with Tax, we raised two antisera $\alpha-151 \mathrm{C}$ and $\alpha-151 \mathrm{~A}$ in rabbits against synthetic TAX1BP1 peptides conjugated to keyhole limpet hemocyanin. These antibodies specifically recognized TAX1BP1 expressed in cultured human cells. For example, $\alpha-151 \mathrm{C}$ reacted with endogenous TAX1BP1 of $\sim 85 \mathrm{kDa}$ in size in HeLa cells (Fig. 1B, lanes 1 and 3) and in HTLV-1-transformed MT4 cells (lane 4). In addition, $\alpha-151 \mathrm{C}$ was also reactive to TAX1BP1 exogenously expressed from a plasmid transiently transfected into HeLa cells (Fig. 1B, lane 2 compared to lanes 1 and 3). 
Using $\alpha-151 \mathrm{C}$ and a mouse monoclonal antibody against Tax, we performed reciprocal coimmunoprecipitation and immunoblotting experiments to characterize the interaction of TAX1BP1 and Tax in cultured cells. Indeed, either $\alpha$-Tax or $\alpha-151 \mathrm{C}$ was able to precipitate both Tax and TAX1BP1 proteins from Tax-expressing HeLa cells (Fig. 1B, lane 3) and HTLVtransformed MT4 cells derived from T lymphocytes (lane 4). By contrast, the formation of TaxTAX1BP1 complex was not seen in mock- or TAX1BP1-transfected HeLa cells (Fig. 1B, lanes 1 and 2), which did not express Tax. Additionally, we also confirmed the interaction between TAX1BP1 and Tax using GST pull-down assay. Here, purified polyhistidine-tagged Tax protein (His-Tax) bound to GST-TAX1BP1 resin (Fig. 1C, lane 4), but not to GST (lane 2). Notably, our data on the interaction of TAX1BP1 and Tax are consistent with findings from yeast two-hybrid and mass spectrometric analyses of Tax-associated proteins in HTLV-1-transformed MT2 and C8166 lymphoblastic cells $(37,38)$.

We mapped the Tax-binding domain in TAX1BP1 to amino acid residues 184-445 (M4; Fig. 1D). We defined this domain based on the Tax-binding activities of 7 TAX1BP1 mutants initially in yeast two-hybrid assays which were subsequently verified by GST pull-downs. The expression of the three mutants that were unable to bind with either Tax or TAX1BP1 was verified by Western blotting (Fig. 1D, inset). In both yeast two-hybrid assays and GST-pull down experiments, we discovered that TAX1BP1 also forms a homodimer. Thus, TAX1BP1's self-dimerization domain resides within amino acid residues 446-600 (M7; Fig. 1D).

Co-localization of TAX1BP1 with Tax and PML. To check that Tax and TAX1BP1 would interact intracellularly, we next queried the subcellular localization of TAX1BP1 using $\alpha-151 \mathrm{C}$ and $\alpha-151 \mathrm{~A}$ (Fig. 2A). We stained HeLa cells with either $\alpha-151 \mathrm{C}$ or $\alpha-151 \mathrm{C}$ affinity-purified using the immunizing peptides (Fig. 2A, panels 1 and 4). Cells were also co-stained for $\alpha$ - 
tubulin, in order to orient the general cellular morphology (Fig. 2A, panels 2, 5 and 8). We observed that cell-endogenous TAX1BP1 was concentrated into intranuclear speckles (Fig. 2A, panels 1 and 3). In support of the specificity of this TAX1BP1 staining, the speckles disappeared when cells were stained with $\alpha-151 \mathrm{C}$ pre-absorbed with the immunizing peptide (Fig. 2A, panels 4 and 6). As confirmation of the staining pattern, HeLa cells visualized using $\alpha-151$ A provided a TAX1BP1-pattern like that observed with $\alpha-151 \mathrm{C}$ (Fig. 2A, compare panel 7 to panel 1).

To assess the localization of TAX1BP1 expressed from a plasmid, we stained HeLa cells transiently transfected with an expression vector for HA-tagged TAX1BP1. Generally in keeping with staining pattern of endogenous TAX1BP1, an anti-HA antibody reacted with TAX1BP1 speckles in transfected cells (Fig. 2B, panel 2). Consistent with results from Western blot analysis in which $\alpha-151 \mathrm{C}$ specifically recognized both endogenous and transfected TAX1BP1 (Fig. 1B), the intranuclear speckles bound to anti-HA overlapped substantially $(84.3 \pm 4.5 \%)$ with those reactive to $\alpha-151 \mathrm{C}$ (Fig. 2B, panels 1 and 3).

Above we demonstrated that TAX1BP1 localizes to intranuclear speckles (Fig. 2). Because Tax was also previously found in intranuclear speckles $(7,39,40)$, we next asked whether TAX1BP1 and Tax might appear together intracellularly, and how one might affect the localization of the other.

When we co-stained Tax-expressing cells for TAX1BP1 and Tax, we noted that both appeared in the nucleus and were concentrated in speckles (Fig. 3A, panels 1 and 2). As indicated by computer-assisted co-localization study, there was $89.1 \pm 5.2 \%$ overlap between Tax-containing speckles and the TAX1BP1 dots (Fig. 3A, panel 3). Thus, TAX1BP1 colocalized with Tax to the same intranuclear structure. Interestingly, the localization of TAX1BP1 
did not change noticeably upon the expression of Tax (Fig. 3A, panel 1, compared to Fig. 2A, panel 1).

The Tax- and TAX1BP1-containing speckles were similar to PML oncogenic domains in appearance. In addition, PML has been shown to modulate Tax function in different contexts (7, 41). However, previous findings on the co-localization of Tax with PML remain controversial. While one group has suggested the residence of Tax in PML domains (7), others have also found that Tax and PML are separate (41). To clarify this issue, we co-stained Tax-expressing cells for Tax and PML. Fig. 3B indicates that the Tax-containing speckles were similar but distinct from PML domains. Hence, there was $48.5 \pm 6.0 \%$ co-localization between Tax and PML (Fig. 3B, panel 3). Likewise, the TAX1BP1 speckles were not identical with the PML dots (Fig. 3C). As such, the two entities only overlapped partially $(41.3 \pm 8.5 \%)$. Thus, our results are consistent with the notion that TAX1BP1 co-localizes substantially with Tax and partially with PML.

Evidence for coactivator function of TAX1BP1. We found unexpectedly that TAX1BP1 fused to Gal4 DNA binding domain (GalBP1) potently activated transcription in yeast. This prompted us to investigate the transcriptional activity of TAX1BP1 in mammalian cells by cotransfecting GalBP1 and a CAT reporter responsive to trans-activators containing Gal4 DNA binding domain (Fig. 4). Indeed, when the expression levels of GalBP1 and GalTax were comparable in HeLa cells as verified by confocal microscopy (data not shown), GalBP1 activated transcription in a dose-dependent manner (Fig. 4B, columns 4-6) with a potency that resembles GalTax, a previously characterized transactivator (Fig. 4A, compare lane 3 to lane 2; Fig. 4B, compare column 4 to column 3). As expected, activation by GalVP16 was even more potent (Fig. 4B, column 2 compared to columns 3 and 4). Based on the activity profiles of four TAX1BP1 mutants (Fig. 4A), the activation domain in TAX1BP1 was mapped to residues 1-326. 
Next we sought to determine the transcriptional activity of TAX1BP1 on Tax-responsive enhancers including the HTLV-1 LTR, אB motif, serum response element (SRE) and cellular cAMP response element (CRE). Because CoCoA is significantly homologous to TAX1BP1 and has been characterized to be a coactivator for NR-dependent transcription (24), which is regulated by Tax (7), we also tested the activity of TAX1BP1 on glucocorticoid response element (GRE). Since the activity of Tax and other coactivators such as CoCoA, GR and GRIP on GRE is known to be activated in the presence of steroids $(7,24)$, dexamethasone was used in some of our experiments. Interestingly, while the expression levels of TAX1BP1 in different groups of cells were similar as verified by Western blotting (Fig. 4C, inset), TAX1BP1 stimulated GRE-dependent luciferase expression, but had no effect on HTLV-1 LTR, אB element, SRE or CRE (Fig. 4C, group 5 compared to groups 1-4). Furthermore, stimulation of GRE by TAX1BP1 occurred in a dose-dependent manner in cells (see the inset in Fig. 4D for verification of the expression levels of TAX1BP1) independent of treatment with dexamethasone (Fig. 4D, groups 1-4). Since CoCoA interacts with GRIP1 and functions as a secondary coactivator in the presence of GR and GRIP1 (24), we asked whether TAX1BP1 might have similar activity. Indeed, TAX1BP1 was able to further activate GRE-driven transcription in the presence of coactivators GR and GRIP1 (Fig. 4D, group 6 compared to group 5). Thus, TAX1BP1 is not only structurally related to but also functionally analogous with CoCoA; and both act as transcriptional coactivator for NRs.

Tax inhibits the transcriptional activity of TAX1BP1. Earlier in this study, we demonstrated the interaction and co-localization of Tax and TAX1BP1 (Fig. 1 and Fig. 3). We also showed the coactivator activity of TAX1BP1 (Fig. 4). To shed light on the functional implications of the interaction between Tax and TAX1BP1, we considered two possibilities. 
First, as a transcriptional coactivator, TAX1BP1 might modulate Tax-dependent transcriptional activity. In particular, Tax could recruit TAX1BP1 to Tax-responsive promoters to effect transcriptional activation. Second, Tax might also impact the cellular function of TAX1BP1.

To investigate these two possibilities, we made use of GalTax and GalBP1; both potently activate transcription from a minimal promoter containing multiple Gal4-binding elements. We queried for the influence of TAX1BP1 and Tax by reciprocally increasing the dose of the indicated expression plasmids. In the case of GalTax, transcriptional activity was totally unaffected when we increased the dose of TAX1BP1 plasmid progressively (Fig. 5A, groups 14; see the inset for verification of the expression levels of TAX1BP1). In sharp contrast, a progressive increase in the dose of Tax plasmid led to significantly reduced GalBP1-dependent activation of CAT expression (Fig. 5A, group 5-8, $\square$; see the inset for verification of the expression levels of Tax). As a control, expression of Tax did not affect the activity of GalVP16 (Fig. 5A, group 5-8, -). Hence, Tax can specifically repress the transcriptional activity of TAX1BP1.

Next we examined the influence of TAX1BP1 on the activity of Tax on the HTLV-1 LTR, $\kappa B$ element and GRE. As expected, Tax potently activated transcription from HTLV-1 LTR and $\kappa \mathrm{B}$ element (Fig. 5B, groups 1 and 2). Also in agreement with previous reports $(7,42)$, Tax repressed GRE-dependent transcription (Fig. 5B, group 3). Notably, the overexpression of TAX1BP1 did not influence Tax activation of HTLV-1 LTR or NFKB, but relieved the repression of GRE-mediated transcription by Tax (Fig. 5B, group 3 compared to groups 1 and 2). On the other hand, compared to TAX1BP1 alone (Fig. 4C, group 5), the expression of Tax eliminated the activation of GRE by TAX1BP1 (Fig. 5B, group 3). 
Because the above experiments were conducted in transfected HeLa cells in which Tax and TAX1BP1 were transiently overexpressed, we sought to verify our findings in suspension $\mathrm{T}$ lymphocytes physiologically infected with HTLV-1. As a first step, we asked whether TAX1BP1 and Tax ambiently expressed in HTLV-1-transformed MT4 cells (Fig. 1B) could be found in the GR-containing protein complex, which is known to mediate GRE-dependent transcription in response to hormone stimulation (21). We performed co-immunoprecipitation with rabbit antiGR antibodies and extracts of MT4 cells. The presence of GR in the immunoprecipitates was confirmed by Western blotting with a mouse anti-GR antibody (Fig. 5C). However, neither TAX1BP1 nor Tax was found in the GR-containing complexes obtained from untreated or dexamethasone-treated MT4 cells (Fig. 5C, lanes 1 and 2). In contrast, we were able to detect TAX1BP1 in the GR-containing complex from Tax-non-expressing Jurkat cells treated with dexamethasone, but not from untreated Jurkat cells (Fig. 5C, lane 4 compared to lane 3). Thus, TAX1BP1 is recruited to the GR-containing complex in Jurkat cells in a manner that depends on dexamethasone stimulation. This mechanism was abrogated in Tax-expressing MT4 cells leading to the absence of TAX1BP1 in the complex that mediates GRE-dependent transcription.

To confirm our findings from co-immunoprecipitation, we assessed the biological effects of Tax and TAX1BP1 in JPX9 T-lymphocytes, in which the expression of Tax was driven by an inducible metallothionein promoter (26). The expression of TAX1BP1 and Tax in mock- and $\mathrm{CdSO}_{4}$-induced JPX9 cells was first confirmed by Western blot analysis (Fig. 5D, insets). Significant less GRE transcriptional activity was observed in JPX9 cells induced to express Tax than in mock-induced JPX9 cells (Fig. 5D, column 2 compared to column 1). In addition, enforced overexpression of TAX1BP1 in induced JPX9 cells led to the restoration of GRE activity to a level comparable to that of mock-induced JPX9 cells (Fig. 5D, column 3 compared 
to columns 1 and 2). These results suggested that TAX1BP1 expressed in induced JPX9 cells was inactivated for transcriptional coactivation, likely through interaction with Tax.

Finally, to establish the role of Tax $1 \mathrm{bp} 1$ in Tax modulation of NR-dependent transcription, we tested the transcriptional activity of Tax in mouse embryonic fibroblasts (MEF) derived from Tax1bp1-disrupted mice (Fig. 6). We first perform Western blotting (Fig. 6A) to assess the expression of Tax $1 \mathrm{bp} 1$ and $\alpha$-tubulin in MEF cells derived from wild type $\left(\mathrm{Tax} 1 \mathrm{bp} 1^{+/+}\right)$, heterozygous (Tax $\left.1 \mathrm{bp} 1^{+-}\right)$and homozygous (Tax $1 \mathrm{bp} 1^{-/}$) Tax $1 \mathrm{bp}-\mathrm{knockout}$ mice, as well as in Tax $1 \mathrm{bp} 1^{-/-}$cells transfected with an expression plasmid for recombinant mouse Tax $1 \mathrm{bp} 1$ (rTax1bp1). The complete loss of Tax1bp1 expression in Tax $1 \mathrm{bp} 1^{-/-}$mice was verified (Fig. 6A, lane 3). We then compared the influence of Tax on the activation of HTLV-1 LTR (Fig. 6B) and GRE (Fig. 6C). We noted that the status of Tax $1 \mathrm{bp} 1$ did not affect the stimulation of LTR by Tax (Fig. 6B). In contrast, the ability of Tax to repress GRE-dependent transcription was lost in Tax $1 b p 1^{-/-}$cells, but not in the other three groups of cells in which Tax $1 b p 1$ is abundantly expressed (Fig. 6C, group 3 compared to groups 1, 2 and 4). Thus, Tax 1 bp1 is specifically required for Tax modulation of NR signaling. 


\section{Discussion}

In this study, we have identified and characterized TAX1BP1, a novel cellular protein that binds to HTLV-1 oncoprotein Tax. TAX1BP1 is a ubiquitously expressed coiled-coil protein, and it uses separate domains to interact with Tax and to form homodimers (Fig. 1). Intracellularly, TAX1BP1 concentrates in Tax-containing intranuclear speckles that partially overlap with PML (Fig. 2 and Fig. 3). Functionally, TAX1BP1 is a transcriptional coactivator for NRs (Fig. 4). While TAX1BP1 does not influence Tax activation of HTLV-1 LTR and NFkB, the expression of Tax inhibits TAX1BP1-mediated transcription in a dose-dependent manner (Fig. 5). In addition, while overexpression of TAX1BP1 in HeLa and HTLV-1-transformed cells causes a relief of Tax-induced repression of NR signaling (Fig. 5), disruption of Tax1bp1 in mouse cells abolished the repressive activity of Tax on NR (Fig. 6). Taken together, our findings suggest that Tax might inhibit NR-dependent transcription by targeting TAX1BP1.

Tax targets a group of coiled-coil proteins. We have previously shown that Tax recognizes a particular coiled-coil structure shared by eight Tax-binding proteins including GPS2, MAD1, IKK- $\gamma$, TAX1BP1 and TAX1BP2 $(23,26,27,36,43)$. The characterization of TAX1BP1 provides new information on the properties of these coiled-coil proteins. Similar to MAD1, TAX1BP1 uses a coiled-coil domain to mediate its interaction with Tax (Fig. 1). In addition, TAX1BP1 is also capable of homodimerization. However, the dimerization domain of TAX1BP1 within the coiled-coil region is separable from the Tax-binding domain (Fig. 1). In other words, different coiled-coil subdomains of TAX1BP1 are responsible for Tax-binding and dimerization. This corroborates the notion that the coiled-coil domains in these proteins are for protein-protein interactions. 
Newly identified Tax-binding proteins such as tropomyosin 5 and TORC1/2/3 also have a coiled-coil domain $(17,18,38)$. In the case of TORC1/2/3, the Tax-binding domain is also within the coiled-coil region (17). Understandably, more Tax-binding coiled-coil proteins will be identified with the advent of new methods for proteomic analysis of Tax partners. We have previously identified IKK- $\gamma$ as a new binding partner of Tax (27) based on sequence similarity to Tax-binding protein TAX1BP2 $(36,43)$. Since CoCoA is significantly homologous to TAX1BP1 and acts as a coactivator for NR-mediated transcription (24), it will be of particularly great interest to elucidate whether CoCoA might also interact directly with Tax.

Physiological function of TAX1BP1. One salient finding that emerges from our study is that TAX1BP1 can function as a transcriptional coactivator for NRs (Fig. 4). NRs are transcription factors that regulate various processes including cell growth and proliferation (21). NR coactivators are a large family of cellular factors recruited by NRs to complement their function in the activation of target genes (22). These coactivators could be broadly divided into three groups that are chromatin-remodeling factors, acetyltransferases and adaptors to basal transcription machinery. Well-known examples of coactivators include SWI/SNF, p160, CBP/p300 and TRAP/DRIP (21). CoCoA homologous to TAX1BP1 has been shown to interact specifically with the N-terminal region of p160 coactivators such as GRIP1 (24), which also localizes to PML oncogenic domains (44). We noted that GRE-dependent transcription was not significantly diminished in Tax1bp1-knockout cells (Fig. 6C). In that regard, other coactivators such as CoCoA might fulfill a redundant function in the absence of TAX1BP1. Nevertheless, additional experiments are required to determine whether TAX1BP1 directly interacts with CoCoA and GRIP1. It will also be of importance to investigate the mechanisms by which TAX1BP1 and CoCoA activate transcription. 
Consistent with the coactivator function of TAX1BP1 for nuclear receptors (Fig. 4), we demonstrated that TAX1BP1 was recruited to the GR-containing protein complex in a hormonedependent manner (Fig. 5). The prevention of this recruitment in HTLV-1-transformed MT4 cells provides one explanation for Tax-induced inhibition of TAX1BP1 function. Existing evidence suggests that Tax directly interacts with TAX1BP1 (Fig. 1) leading to its dissociation from the GR-containing complex critically involved in transcriptional activation (Fig. 5). Further elucidation of the direct protein partner(s) of TAX1BP1 in that complex will derive important mechanistic insight into the roles of TAX1BP1 in transcription.

Our work does not preclude TAX1BP1 from other functions beyond transcriptional regulation. Previous studies have revealed several other binding partners of TAX1BP1 including A20, TRAF6 and Chfr $(23,34,45)$. TAX1BP1 may also have cell cycle regulatory activity (our unpublished data). Although A20, TRAF6 and Chfr serve different functions in regulating $\mathrm{NF \kappa B}$, apoptosis, interleukin-1 signaling and mitotic progression, more recent studies suggest that all three have ubiquitin ligase and/or deubiquitinase activities (46-48). In addition, the ubiquitination of Tax has also been demonstrated (49). In light of this, we speculate that TAX1BP1 might be involved in some aspect of protein ubiquitination. Elsewhere TAX1BP1 is shown to be a repressor of TRAF6-mediated NFKB activation; and Tax $1 \mathrm{bp}^{-{ }^{--}}$mice exhibit a hyper-inflammatory phenotype when exposed to interleukin-1, lipopolysaccharide or tumor necrosis factor $\alpha$ (Iha et al., in preparation). Thus TAX1BP1 may provide different adaptor roles and contributes different function depending on the protein-protein complexes into which it intercalates. Full clarification of TAX1BP1's functions awaits further investigation.

Tax- and TAX1BP1-containing intranuclear speckles. The subcellular localization of Tax has been well documented (39). Compared to the existing model, two additional points revealed 
in our study are noteworthy. First, TAX1BP1 is a component of Tax-containing intranuclear speckles (Fig. 2 and Fig. 3). Second, these Tax- and TAX1BP1-containing speckles partially overlap with the PML oncogenic domains, also known as nuclear domains 10 (ND10) in the literature (Fig. 3).

In support of the partial overlapping of the PML domains with speckles containing Tax and TAX1BP1, several proteins that are known to interact with Tax or TAX1BP1 have also been found in the PML domains. These include the CBP coactivator, p53, Chfr and HDACs (50). PML domains have been suggested to play a role in transcriptional control, tumor suppression, DNA repair, protein degradation and apoptosis (50). All these functions are highly relevant to Tax. Plausibly, the overlapping of Tax/TAX1BP1 with PML domains may provide an opportunity for their interaction with other components in that compartment.

A model for Tax repression of NR signaling. We have presented several lines of evidence that support a new model for Tax repression of NR signaling. First, TAX1BP1 is a component of the NR-containing protein complex (Fig. 5) and it serves a transcriptional coactivator function for NRs (Fig. 4). Second, Tax interacts specifically with TAX1BP1 (Fig. 1). Third, the expression of Tax leads to dissociation of TAX1BP1 with NRs and diminution of NR-dependent transcription (Fig. 5). Finally, while the expression of TAX1BP1 relieves the inhibition of Tax repression of NR activity (Fig. 5), genetic knockout of Tax $1 \mathrm{bp} 1$ in mice abrogates the repressive activity of Tax on NR (Fig. 6).

The above four lines of evidence are consistent with a model in which Tax interacts with and inhibits TAX1BP1 to affect NR activity. This new model explains the previous findings that Tax represses the transcriptional activity of NRs $(7,42)$. 


\section{Acknowledgments}

Grant support: Concern Foundation research grant (2002) and grant HKU 7683/05M from Hong Kong Research Grants Council.

We thank H. Gronemeyer and K. R. Yamamoto for gifts of plasmid. 


\section{References}

1. Matsuoka M. Human T-cell leukemia virus type I (HTLV-I) infection and the onset of adult T-cell leukemia (ATL). Retrovirology 2005;2:27.

2. Grassmann R, Aboud M, Jeang KT. Molecular mechanisms of cellular transformation by HTLV-1 Tax. Oncogene 2005;24:5976-85.

3. Yoshida M. Multiple viral strategies of HTLV-1 for dysregulation of cell growth control. Annu Rev Immunol 2001;19:475-96.

4. Jeang KT, Widen SG, Semmes OJ, Wilson SH. HTLV-I trans-activator protein, Tax, is a trans-repressor of the human $\beta$-polymerase gene. Science 1990;247:1082-4.

5. Pise-Masison CA, Rodonovich M, Sakaguchi K, Appella E, Brady J. Phosphorylation of p53: a novel pathway for p53 inactivation in human T-cell lymphotropic virus type 1transformed cells. J Virol 1998;72:6348-55.

6. Van PL, Yim KW, Jin DY, Chen DJ, Jeang KT. Genetic evidence of a role for ATM in functional interaction between HTLV-I Tax and p53. J Virol 2001;75:396-407.

7. Doucas V, Evans RM. Human T-cell leukemia retrovirus-Tax protein is a repressor of nuclear receptor signaling. Proc Natl Acad Sci USA 1999;96:2633-8.

8. Kibler KV, Jeang KT. CREB/ATF-dependent repression of cyclin A by human T-cell leukemia virus type 1 Tax protein. J Virol 2001;75:2161-73.

9. Nicot C, Mahieux R, Pise-Masison C, et al. Human T-cell lymphotropic virus type 1 Tax represses c-Myb-dependent transcription through activation of the $\mathrm{NF}-\kappa \mathrm{B}$ pathway and modulation of coactivator usage. Mol Cell Biol 2001;21:7391-402.

10. Riou P, Bex F, Gazzolo L. HTLV-1 Tax represses MyoD-dependent transcription by inhibiting MyoD-binding to the KIX domain of p300. J Biol Chem 2000;275:10551-60. 
11. Arnulf B, Villemain A, Nicot C, et al. Human T-cell lymphotropic virus oncoprotein Tax represses TGF- $\beta 1$ signaling in human T cells via c-Jun activation: a potential mechanism of HTLV-I leukemogenesis. Blood 2002;100:4129-38.

12. Tie F, Adya N, Greene WC, Giam CZ. Interaction of the human T-lymphotropic virus type 1 Tax dimer with CREB and the viral 21-base-pair repeat. J Virol 1996;70:8368-74.

13. Jin DY, Jeang KT. HTLV-I Tax self-association in optimal trans-activation function. Nucl Acids Res 1997; 25:379-88.

14. Kimzey AL, Dynan WS. Specific regions of contact between human T-cell leukemia virus type I Tax protein and DNA identified by photocross-linking. J Biol Chem 1998;273: $13768-75$.

15. Ching YP, Chun AC, Chin KT, Zhang ZQ, Jeang KT, Jin DY. Specific TATAA and bZIP requirements suggest that HTLV-I Tax has transcriptional activity subsequent to the assembly of an initiation complex. Retrovirology 2004;1:18.

16. Kwok RP, Laurance ME, Lundblad JR, et al. Control of cAMP-regulated enhancers by the viral transactivator Tax through CREB and the co-activator CBP. Nature 1996;380:642-6.

17. Koga H, Ohshima T, Shimotohno K. Enhanced activation of Tax-dependent transcription of human T-cell leukemia virus type I (HTLV-I) long terminal repeat by TORC3. J Biol Chem 2004;279:52978-83.

18. Siu YT, Chin KT, Siu KL, Choy EYW, Jeang KT, Jin DY. TORC1 and TORC2 coactivators are required for Tax activation of the human T-cell leukemia virus type 1 long terminal repeats. J Virol 2006;80:7052-9. 
19. Uittenbogaard MN, Armstrong AP, Chiaramello A, Nyborg JK. Human T-cell leukemia virus type I Tax protein represses gene expression through the basic helix-loop-helix family of transcription factors. J Biol Chem 1994;269:22466-9.

20. Suzuki T, Uchida-Toita M, Yoshida M. Tax protein of HTLV-1 inhibits CBP/p300mediated transcription by interfering with recruitment of CBP/p300 onto DNA element of E-box or p53 binding site. Oncogene 1999;18:4137-43.

21. Gronemeyer H, Gustafsson JA, Laudet V. Principles for modulation of the nuclear receptor superfamily. Nat Rev Drug Discov 2004;3:950-64.

22. Fu M, Wang C, Zhang X, Pestell RG. Acetylation of nuclear receptors in cellular growth and apoptosis. Biochem Pharmacol 2004;68:1199-208.

23. DeValck D, Jin DY, Heyninck K, et al. The zinc finger protein A20 interacts with a novel anti-apoptotic protein which is cleaved by specific caspases. Oncogene 1999;18:4182-90.

24. Kim JH, Li H, Stallcup MR. CoCoA, a nuclear receptor coactivator which acts through an N-terminal activation domain of p160 coactivators. Mol Cell 2003;12:1537-49.

25. Yang CK, Kim JH, Li H, Stallcup MR. Differential use of functional domains by coiledcoil coactivator in its synergistic coactivator function with $\beta$-catenin or GRIP1. J Biol Chem 2006;281:3389-97.

26. Jin DY, Spencer F, Jeang KT. Human T-cell leukemia virus type I oncoprotein Tax targets the human mitotic checkpoint protein MAD1. Cell 1998;93:81-91.

27. Jin DY, Giordano V, Kibler KV, Nakano H, Jeang KT. Role of adaptor function in

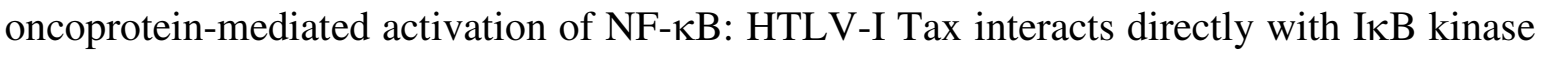

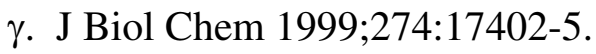


28. Rogatsky I, Wang JC, Derynck MK, et al. Target-specific utilization of transcriptional regulatory surfaces by the glucocorticoid receptor. Proc Natl Acad Sci USA 2003;100: 13845-50.

29. Voegel JJ, Heine MJ, Zechel C, Chambon P, Gronemeyer H. TIF2, a $160 \mathrm{kDa}$ transcriptional mediator for the ligand-dependent activation function AF-2 of nuclear receptors. EMBO J 1996;15:3667-75.

30. Huang GJ, Zhang ZQ, Jin DY. Stimulation of IKK- $\gamma$ oligomerization by the T-cell leukemia virus oncoprotein Tax. FEBS Lett 2002;531:494-8.

31. Chun ACS, Jin DY. Transcriptional regulation of mitotic checkpoint gene MAD1 by p53. J Biol Chem 2003;278:37439-50.

32. Zhou Y, Ching YP, Chun ACS, Jin DY. Nuclear localization of the cell cycle regulator CDH1 and its regulation by phosphorylation. J Biol Chem 2003;278:12530-6.

33. Chin KT, Zhou HJ, Wong CM, et al. The liver-enriched transcription factor CREB-H is a growth suppressor protein underexpressed in hepatocellular carcinoma. Nucl Acids Res 2005;33:1859-73.

34. Ling L, Goeddel DV. T6BP, a TRAF6-interacting protein involved in IL-1 signaling. Proc Natl Acad Sci USA 2000;97:9567-72.

35. Sternsdorf T, Jensen K, Zuchner D, Will H. Cellular localization, expression, and structure of the nuclear dot protein 52. J Cell Biol 1997;138:435-48.

36. Chun ACS, Zhou Y, Wong CM, Kung H, Jeang KT, Jin DY. Coiled-coil motif as a structural basis for the interaction of HTLV type 1 Tax with cellular cofactors. AIDS Res Hum Retrov 2000;16:1689-94. 
37. Gachon F, Peleraux A, Thebault S, et al. CREB-2, a cellular CRE-dependent transcription repressor, functions in association with Tax as an activator of the human T-cell leukemia virus type 1 promoter. J Virol 1998;72:8332-7.

38. Wu K, Bottazzi ME, de la Fuente C, et al. Protein profile of Tax-associated complexes. J Biol Chem 2004;279:495-508.

39. Semmes OJ, Jeang KT. Localization of human T-cell leukemia virus type 1 Tax to subnuclear compartments that overlap with interchromatin speckles. J Virol 1996;70:634757.

40. Haoudi A, Daniels RC, Wong E, Kupfer G, Semmes OJ. Human T-cell leukemia virus-I Tax oncoprotein functionally targets a subnuclear complex involved in cellular DNA damage-response. J Biol Chem 2003;278:37736-44.

41. Ariumi Y, Ego T, Kaida A, Matsumoto M, Pandolfi PP, Shimotohno K. Distinct nuclear body components, PML and SMRT, regulate the trans-acting function of HTLV-1 Tax oncoprotein. Oncogene 2003;22:1611-9.

42. Wienzek S, Dobbelstein M. Viral and cellular factors that target the promyelocytic leukemia oncogenic domains strongly activate a glucocorticoid-responsive promoter. J Virol 2001;75:5391-7.

43. Ching YP, Chan SF, Jeang KT, Jin DY. Retroviral oncoprotein Tax targets coiled-coil centrosomal protein TAX1BP2 to induce centrosome overduplication. Nat Cell Biol 2006;8:717-24.

44. Baumann $\mathrm{CT}$, Ma H, Wolford $\mathrm{R}$, et al. The glucocorticoid receptor interacting protein 1 (GRIP1) localizes in discrete nuclear foci that associate with ND10 bodies and are enriched in components of the 26S proteasome. Mol Endocrinol 2001;15:485-500. 
45. Matsusaka T, Pines J. Chfr acts with the p38 stress kinases to block entry into mitosis in mammalian cells. J Cell Biol 2004;166:507-16.

46. Wertz IE, O'Rourke KM, Zhou H, et al. De-ubiquitination and ubiquitin ligase domains of A20 downregulate NF-кB signalling. Nature 2004;430:694-9.

47. Kang D, Chen J, Wong J, Fang G. The checkpoint protein Chfr is a ligase that ubiquitinates Plk1 and inhibits Cdc2 at the G2 to M transition. J Cell Biol 2002;156:249-59.

48. Sun L, Deng L, Ea CK, Xia ZP, Chen ZJ. The TRAF6 ubiquitin ligase and TAK1 kinase mediate IKK activation by BCL10 and MALT1 in T lymphocytes. Mol Cell 2004;14:289301.

49. Peloponese JM Jr, Iha H, Yedavalli VR, et al. Ubiquitination of human T-cell leukemia virus type 1 Tax modulates its activity. J Virol 2004;78:11686-95.

50. Dellaire G, Bazett-Jones DP. PML nuclear bodies: dynamic sensors of DNA damage and cellular stress. BioEssays 2004;26:963-77. 


\section{Figure Legends}

Figure 1. Interaction between TAX1BP1 and Tax. $A$, schematic diagram of TAX1BP1 and CoCoA. The NDP52-homologous, coiled coil (CC) and zinc finger (ZF) domains are indicated. B, co-immunoprecipitation of Tax and TAX1BP1 from Tax-expressing HeLa cells and HTLV-1transformed MT4 cells. HeLa cells were mock transfected (lane 1) or transfected with TAX1BP1 (lane 2) or Tax (lane 3). Crude cell extracts were analyzed by Western blotting with rabbit antiTAX1BP1 ( $\alpha-151 \mathrm{C})$ or mouse anti-Tax $(\alpha$-Tax). Cell lysates were also immunoprecipitated (IP) with either mouse $\alpha$-Tax or rabbit $\alpha$-151C. The $\alpha$-Tax precipitate was blotted with $\alpha-151 \mathrm{C}$ and rabbit $\alpha$-Tax. The $\alpha$-151C precipitate was blotted with mouse $\alpha$-Tax and another rabbit antiserum against TAX1BP1 ( $\alpha-151 \mathrm{~A}) . C$, GST pull-down assay. GST (lanes 1 and 2) and GSTTAX1BP1 (GST-BP1; lanes 3 and 4) were bound to Sepharose resins. Resins were incubated with His-Tax, and bound proteins were then eluted. Flow-through (FT, lanes 1 and 3) and eluates (lanes 2 and 4) were analyzed by Western blotting with mouse $\alpha$-Tax. $D$, mapping of Taxbinding and dimerization domains in TAX1BP1. Interactions between the indicated pairs of proteins were defined by yeast two-hybrid analysis and scored by fold-activation in $\beta$ galactosidase ( $\beta$-gal) activity. The interactions were further confirmed by in vitro affinity binding assay (data not shown). Expression of M1, M3 and M5 mutants fused to Gal4 activation domain (AD-M1/3/5) was verified by Western blotting with anti-AD (inset).

Figure 2. Subcellular localization of TAX1BP1 protein in cultured cells. $A$, localization of endogenous TAX1BP1. HeLa cells were stained with purified $\alpha-151 \mathrm{C}$ (panel 1), $\alpha$-151C preincubated with $3.5 \mu \mathrm{g}$ of immunizing peptide $(151 \mathrm{C}+$ pep.; panel 2$)$, or $\alpha-151 \mathrm{~A}$ (panel 7). Cells 
were co-stained with mouse anti- $\alpha$-tubulin (panels 2, 5 and 8). The TAX1BP1 (red) and $\alpha$ tubulin (green) fluorescent signals were then overlaid by computer assistance (panels 3, 6 and 9). The same fields are shown in panels 1-3, 4-6, and 7-9. Bar, $20 \mu \mathrm{m} . B$, localization of exogenously expressed TAX1BP1. HeLa cells were transfected with pHABP1 and stained with $\alpha-151 \mathrm{C}$ (lane 1) and $\alpha-\mathrm{HA}$ (lane 2). The TAX1BP1 (red; representing endogenous and transfected TAX1BP1) and HA (green; representing transfected TAX1BP1) fluorescent signals were overlaid in panel 3. Co-localizations are shown in yellow. The same fields are shown in panels 1-3. The arrow indicates transfected cell. Results represent three independent experiments and $84.3 \pm 4.5 \%$ co-localization was detected for 150 transfected cells analyzed. Bar, $20 \mu \mathrm{m}$.

Figure 3. Co-localization of TAXBP1 with Tax and PML. A, co-localization of TAX1BP1 with Tax. HeLa cells transiently transfected with Tax-expressing plasmid were co-stained with $\alpha$ 151C (panel 1) and $\alpha$-Tax (panel 2) antibodies. In panel 3, the TAX1BP1 (red) and Tax (green) fluorescent signals were overlaid by computer assistance. Co-localizations are in yellow. The same fields are shown in panels 1-3. Arrows indicate transfected cells. Results are representative of three independent experiments and $89.1 \pm 5.2 \%$ co-localization of the two fluorescent signals was detected for 150 transfected cells analyzed. Bar, $20 \mu \mathrm{m} . B$, co-localization of Tax with PML. HeLa cells transiently transfected with Tax-expressing plasmid were co-stained with $\alpha$-Tax (panel 1) and $\alpha$-PML (panel 2) antibodies. In panel 3, the Tax (red) and PML (green) fluorescent signals were overlaid by computer assistance. Co-localizations are in yellow. The same fields are shown in panels 1-3. Results are representative of three independent experiments and $48.5 \pm$ $6.0 \%$ co-localization was detected for 150 transfected cells analyzed. Bar, $20 \mu \mathrm{m}$. C, colocalization of TAX1BP1 with PML. HeLa cells were co-stained with $\alpha-151 \mathrm{C}$ (panel 1) and $\alpha$ 29 
PML (panel 2) antibodies. In panel 3, the TAX1BP1 (red) and PML (green) fluorescent signals were overlaid by computer assistance. Co-localizations are in yellow. The same fields are shown in panels 1-3. Results are representative of three independent experiments and $41.3 \pm 8.5 \%$ colocalization was detected for 150 cells analyzed. Bar, $20 \mu \mathrm{m}$.

Figure 4. TAX1BP1 is a transcriptional coactivator. $A$, mapping of the trans-activation domain in TAX1BP1. HeLa cells were transiently transfected with $1 \mu \mathrm{g}$ of pG5CAT reporter and $5 \mu \mathrm{g}$ of plasmids expressing Gal4 DNA binding domain (Gal; lane 1), GalTax (lane 2), GalBP1 (lane 3), GalBP1 mutant M8 (GalBP1M8; lane 4) and GalBP1 mutant M9 (GalBP1M9; lane 5). CAT assays were performed 48 hours after transfection. Summarized on the right are transactivation activities of different TAX1BP1 fusion proteins (BP1: GalBP1 wild-type; M8, M9, M1 and M2: GalBP1 mutants). The activity was scored by fold-activation $(+++>10 ; 5<++<10 ; 2<+<5$; - < 2). AcCM: acetyl chloramphenicol. CM: chloramphenicol. B, TAX1BP1 activates transcription in a dose-dependent manner when targeted to a minimal promoter. HeLa cells were transfected with pG5CAT reporter and plasmids expressing the indicated proteins. Decreasing amounts (5, 2 and $1 \mu \mathrm{g}$ ) of GalBP1 plasmids were added to cells (columns 4-6), while $5 \mu \mathrm{g}$ of plasmids expressing all other Gal or Gal fusion proteins were used in the transfection (columns 1, 2, 3, 4, 7 and 8). The expression of Gal and Gal fusion proteins was verified by confocal microscopy. The transfection efficiency was normalized by pSV- $\beta$-Gal. The results are the average from three independent experiments and error bars indicate standard deviations. $C$, coactivator activity of TAX1BP1 on different enhancers. HeLa cells were transfected with luciferase reporter plasmids (100 ng) driven by the indicated enhancer elements. A TAX1BP1 expression plasmid (100 ng) was either given (-; w/ BP1) or not given ( $\square$; w/o BP1) to cells in 
each group. Cells in group 5 were also treated with $1 \mu \mathrm{M}$ dexamethasone for 12 hours before harvest. Dual luciferase assay was performed. Firefly luciferase activity was measured by arbitrary units (au) and normalized to Renilla luciferase activity. The graph represents three independent experiments and similar results were also obtained in Jurkat cells (data not shown). The expression of TAX1BP1 in different groups of cells was verified by Western blotting (inset). $D$, coactivator activity of TAX1BP1 on GRE. Fixed amounts of pGRELuc reporter plasmid (50 ng) and increasing amounts $(0,50 \mathrm{ng}, 150 \mathrm{ng}$ and $450 \mathrm{ng})$ of TAX1BP1 expression plasmid were given to HeLa cells (groups 1-4) treated (-; w/ Dex) or not treated ( $\square$; w/o Dex) with $1 \mu \mathrm{M}$ dexamethasone (Dex). Cells in groups 5 and 6 received pGRELuc reporter (50 ng) and expression plasmids pCDNArGR and pSG5TIF2 (75 ng each) for GR and GRIP. In addition, cells in group 6 were cotransfected with TAX1BP1 expression plasmid (100 ng). Dual luciferase assay was performed as in $C$. Expression of TAX1BP1 in cells (groups 2-4) was verified by Western blotting (inset).

Figure 5. Tax inhibits the coactivator activity of TAX1BP1. A, influence of TAX1BP1 on Tax activity (groups 1-4) and influence of Tax on TAX1BP1 activity (groups 5-8). For groups 1-4, HeLa cells were transfected with pG5CAT reporter $(1 \mu \mathrm{g})$, GalTax plasmid $(5 \mu \mathrm{g})$ and increasing amounts of TAX1BP1 plasmid (0 to $5 \mu \mathrm{g})$. For groups $5-8$, HeLa cells were transfected with pG5CAT reporter $(1 \mu \mathrm{g})$, GalBP1 (口) or GalVP16 (ロ) plasmid $(5 \mu \mathrm{g})$ and increasing amounts of Tax plasmid (0 to $5 \mu \mathrm{g}$ ). Expression of BP1 and Tax in cells (groups 2-4 and groups 6-8, respectively) was verified by Western blotting (insets). $B$, inhibition of GRE activity by Tax can be rescued by overexpression of TAX1BP1. HeLa cells were transfected with luciferase reporter plasmids driven by the indicated enhancer elements. Cells in each group 
received neither Tax nor TAX1BP1 ( $\square$; basal), Tax alone ( $\boxminus$; Tax), or both Tax and TAX1BP1 $(\mathbf{a}$; Tax + BP1). Cells in group 3 were also treated with $1 \mu \mathrm{M}$ dexamethasone for 12 hours before the assay. Dual luciferase assay was performed and normalized luciferase activity (luc. activity) was measured by arbitrary units (au). Similar results were obtained in three independent experiments and also in Jurkat cells. $C$, dissociation of TAX1BP1 from GR-containing complex in HTLV-1-transformed leukemic cells. MT4 and Jurkat (Jkt) T lymphocytes were mock-treated or treated with $1 \mu \mathrm{M}$ dexamethasone for 12 hours. Cell lysates were immunoprecipitated (IP) with rabbit anti-GR. The precipitates were then blotted with rabbit anti-151C, mouse anti-Tax and mouse anti-GR. $D$, overexpression of TAX1BP1 can rescue the inhibition of GRE activity in Tax-expressing cells. JPX9 cells were transfected with pGRELuc reporter alone (groups 1 and 2) or with pGRELuc plus TAX1BP1 expression plasmid (group 3). Cells were treated with $1 \mu \mathrm{M}$ dexamethasone for 12 hours. Cells were mock-induced (group 1) or induced with $25 \mu \mathrm{M} \mathrm{CdSO}_{4}$ for 3 hours (groups 2 and 3). Dual luciferase assay was performed as in $C$ with the same amount of cell lysate from each group. Results represent three independent transfections. Expression of TAX1BP1 and Tax in cells was verified by Western blotting (inset).

Figure 6. Genetic knockout of Taxlbpl abolishes the repression of GRE by Tax. A, expression of Tax1bp1 in different groups of MEF cells. Western blot analysis of Tax1bp1 (BP1) and $\alpha$ tubulin was performed on lysates of MEF cells with wild type Taxlbpl (lane 1), MEF cells with heterozygous deletion of Taxlbpl (lane 2), MEF cells with homozygous deletion of Taxlbpl (lane 3), and Taxlbp1 $1^{-/-}$cells transfected with an expression plasmid for recombinant mouse Tax1bp1 (rTax1bp1, lane 4). B, Tax activation of HTLV-1 LTR in MEF cells. The four groups of cells as described in $A$ were transfected with Tax plasmid and LTR-luciferase reporter. $C$, Tax 
activity on GRE in MEF cells. The four groups of cells as described in $A$ were transfected with Tax plasmid and pGRELuc reporter. Cells were treated with $1 \mu \mathrm{M}$ dexamethasone for 12 hours. Dual luciferase assay was performed and results represent the average normalized luciferase activity in arbitrary units (au) obtained from three independent experiments. 

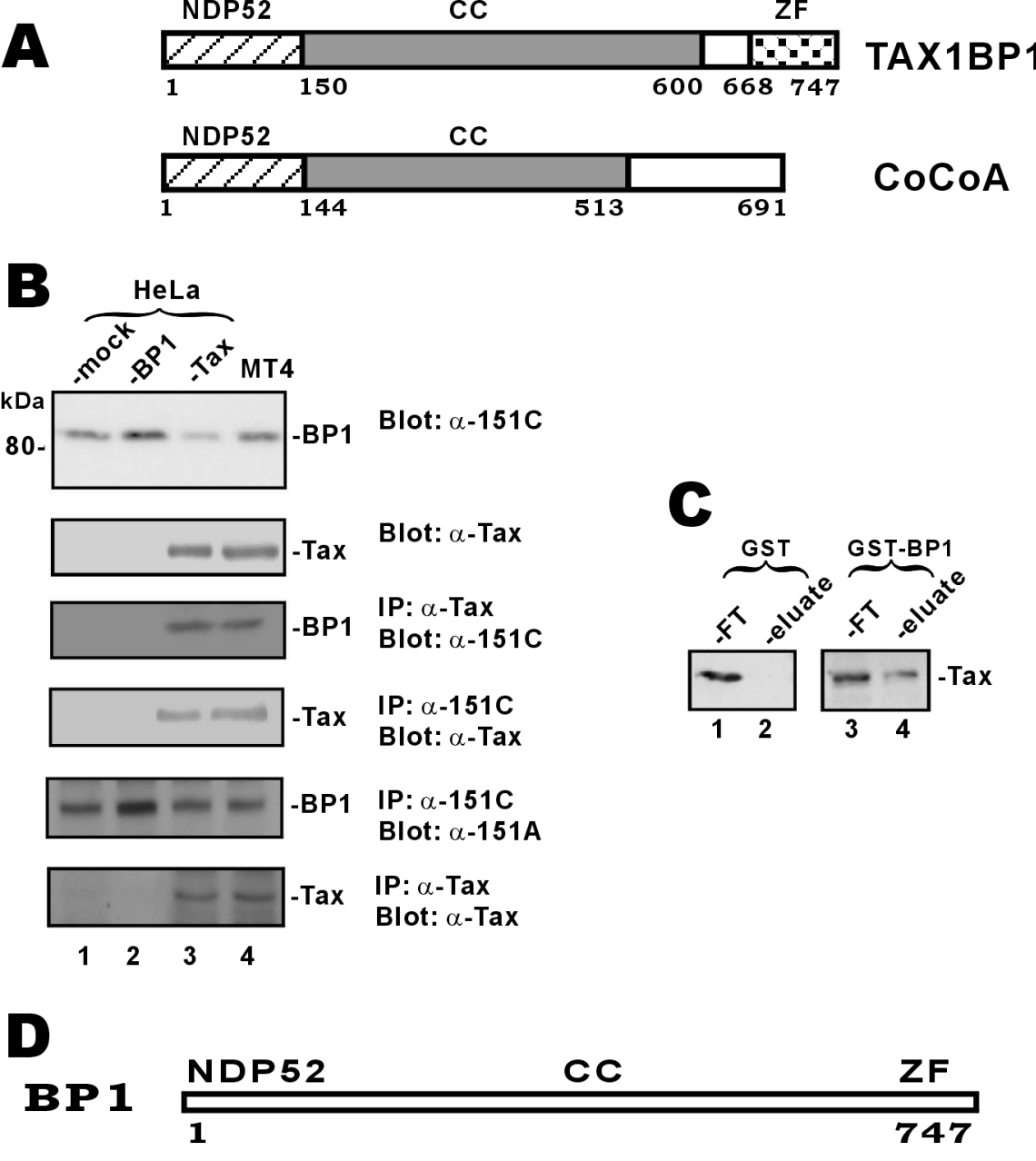

MI 1

MI 2

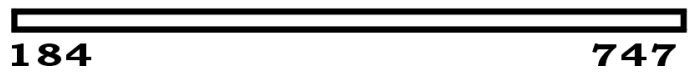

IM 3

M4

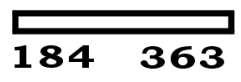

M 5

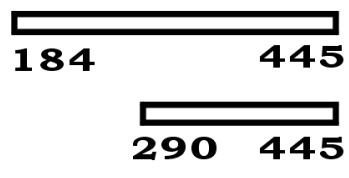

M 6

M 7
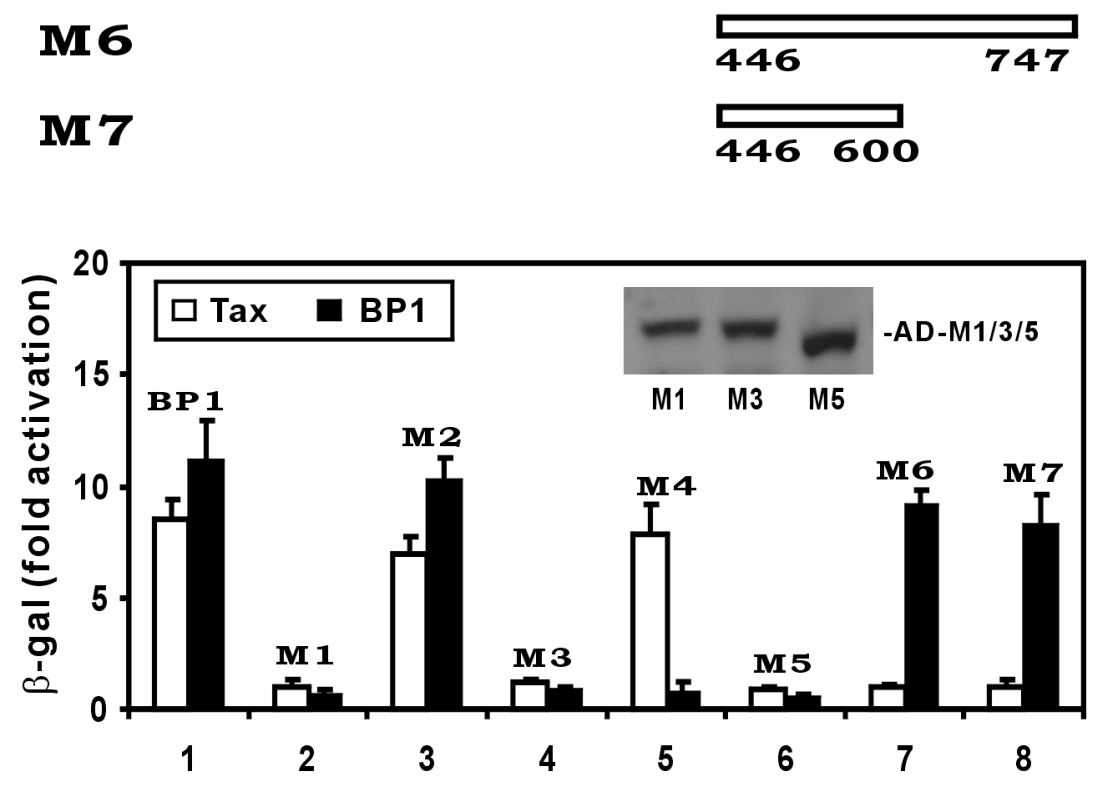
BP1

151C

1

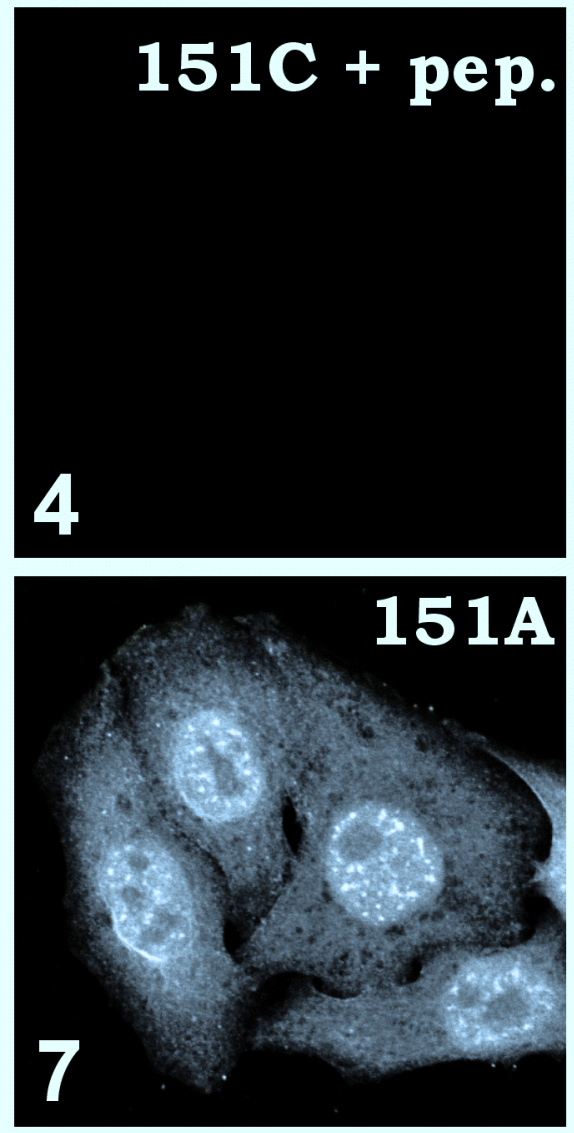

B

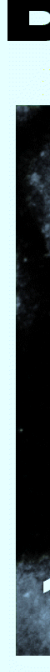

tubulin
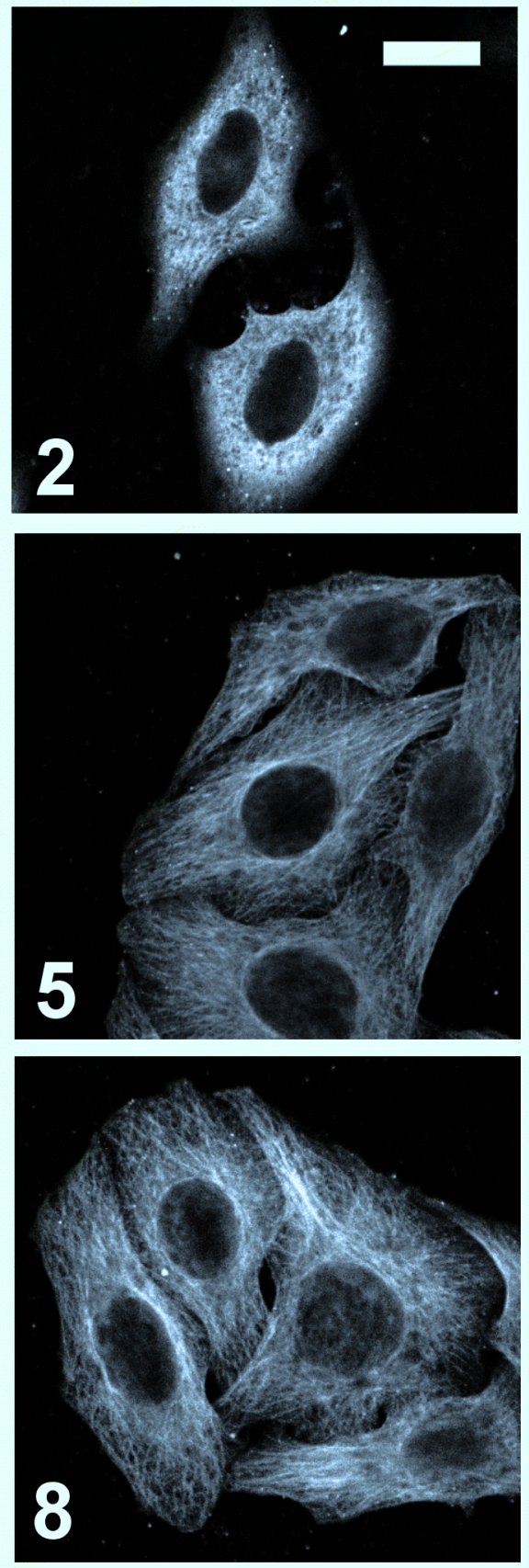

HA

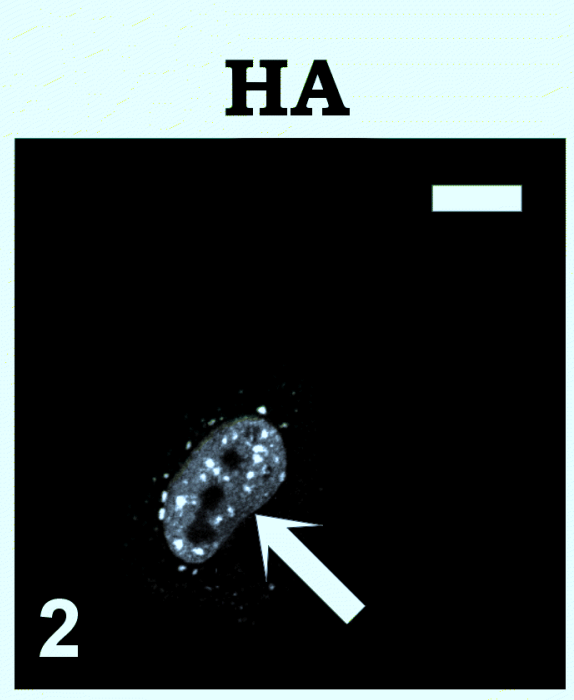

overlay

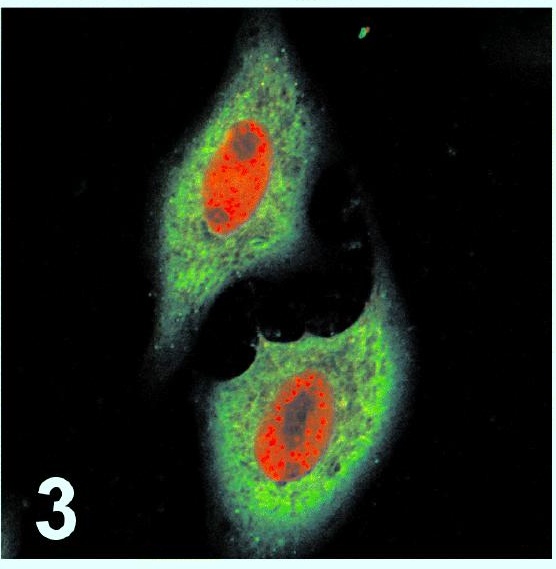

6

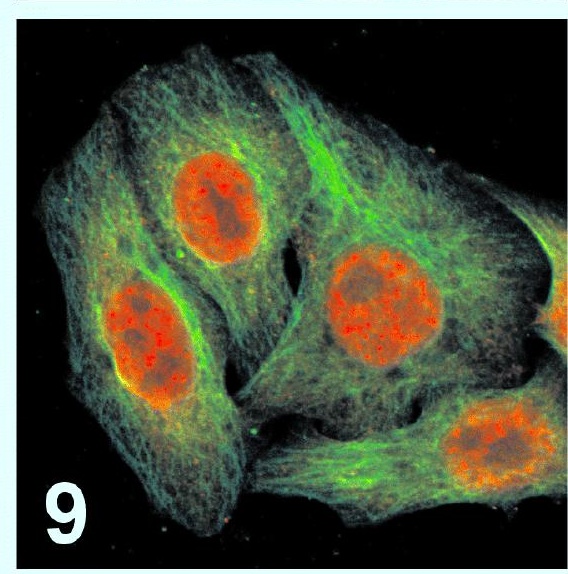

9
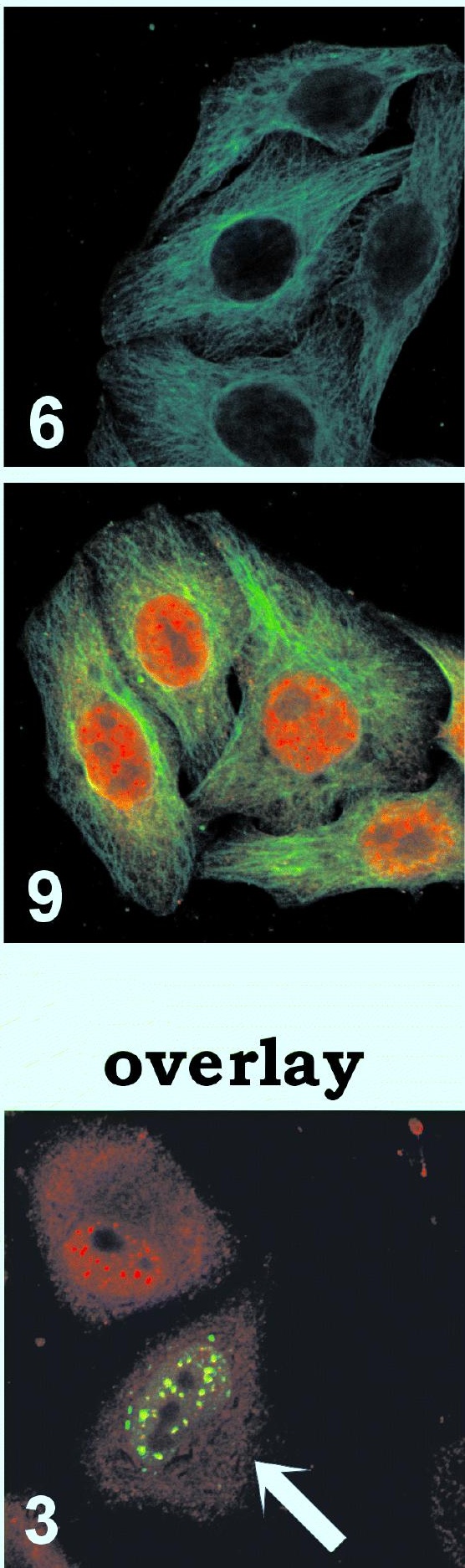

Figure 2 

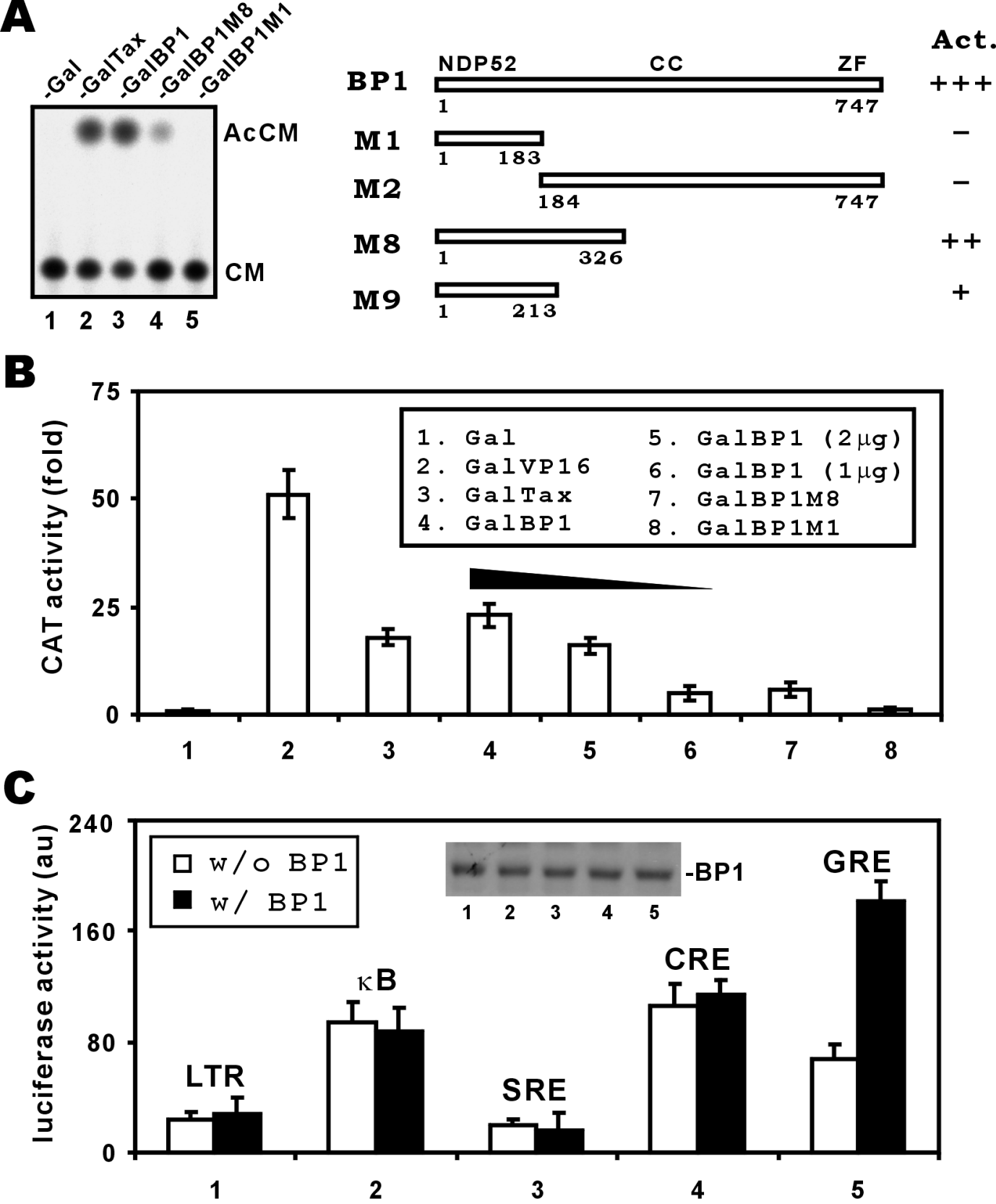

D
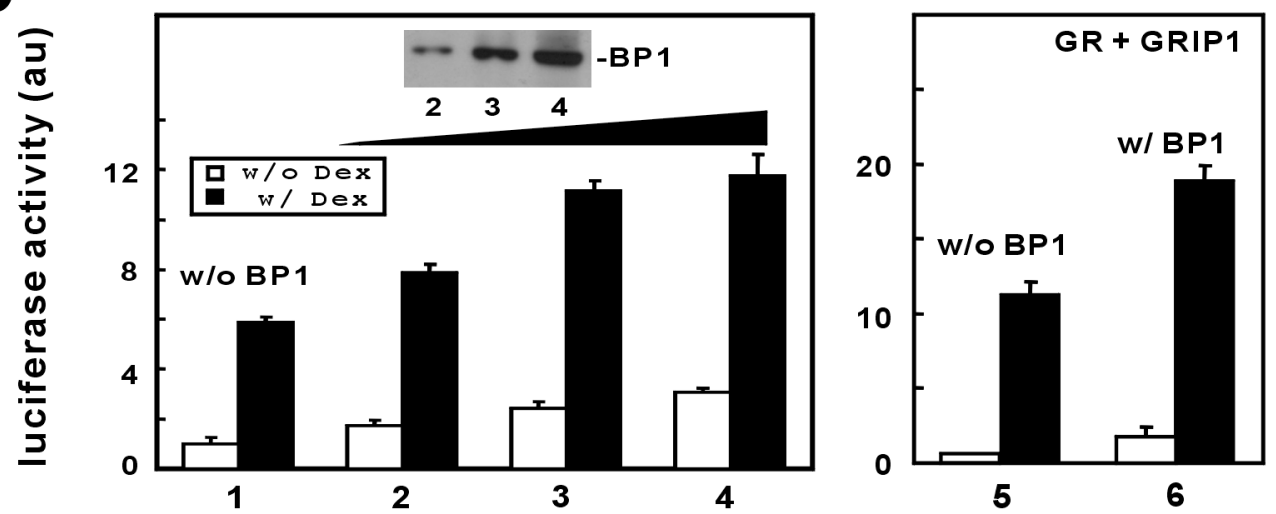

Figure 4 

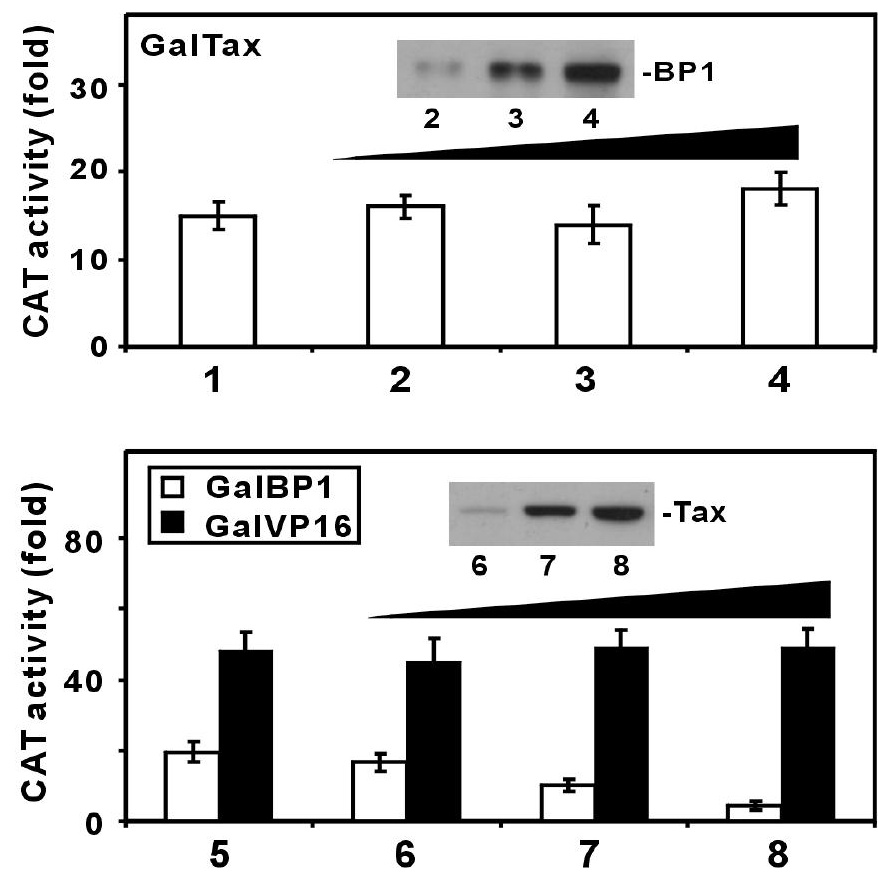

\section{B}
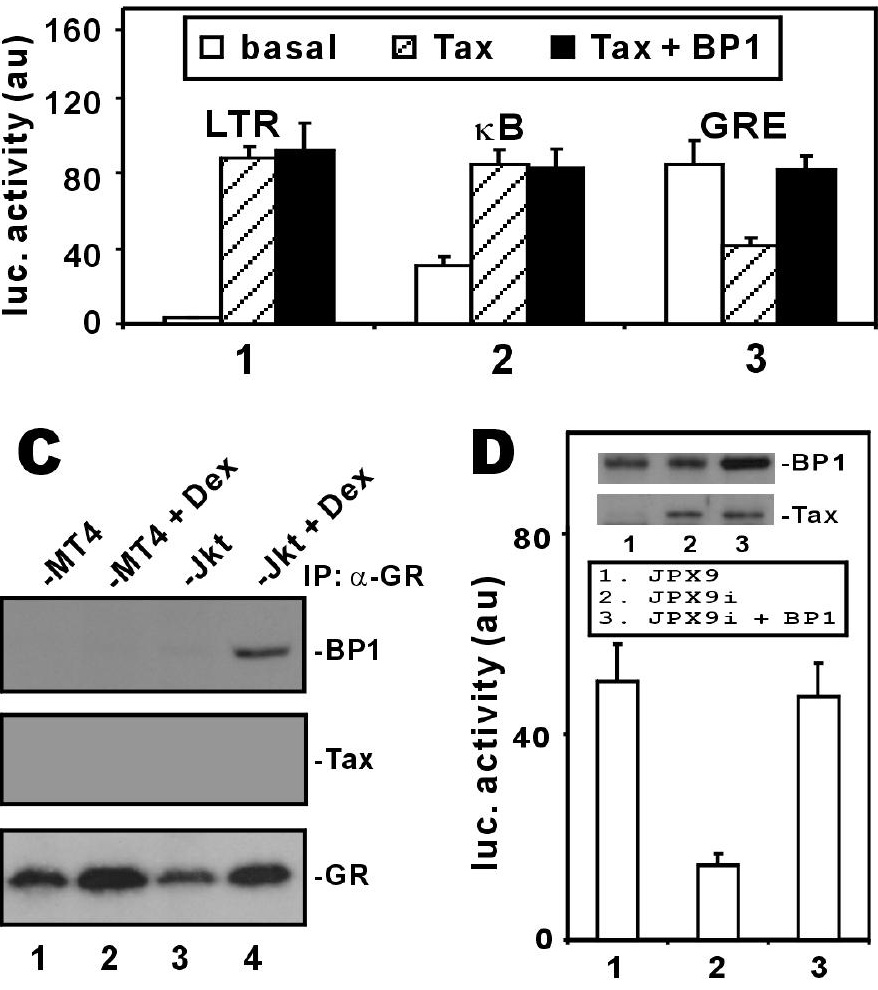
